\title{
Study of corrections to the eikonal approximation
}

\author{
C. Hebborn ${ }^{* 1}$ and P. Capel ${ }^{1,2}$ \\ ${ }^{1}$ Physique Nucléaire et Physique Quantique (CP 229) \\ Université libre de Bruxelles (ULB) \\ B-1050 Brussels, Belgium \\ E-mail: chloe.hebborn@ulb.ac.be \\ ${ }^{2}$ Institut für Kernphysik \\ Technische Universität Darmstadt \\ D-64289 Darmstadt, Germany \\ E-mail: pierre.capel@ulb.ac.be
}

For the last decades, multiple international facilities have developed Radioactive-Ion Beams (RIB) to measure reaction processes including exotic nuclei. These measurements coupled with an accurate theoretical model of the reaction enable us to infer information about the structure of these nuclei. The partial-wave expansion provides a precise description of two-body collisions but has a large computational cost, when extended to two- (or more) body description of the projectile. To cope with this issue, the eikonal approximation is a powerful tool as it reduces the computational time and still describes the quantum effects observed in reaction observables. However, its range of validity is restricted to high energy and to forward scattering angles. In this work, we analyse the extension of the eikonal approximation to lower energies and larger angles through the implementation of two corrections. These aim to improve the treatment of the nuclear and Coulomb interactions within the eikonal model. The first correction is based on an expansion of the $T$-matrix while the second relies on a semi-classical approach. They permit to better account for the deflection of the projectile by the target, which is neglected in the standard eikonal model. The gain in accuracy of each correction is evaluated through the analyses of angular cross sections computed with the standard eikonal model, its corrections and the partialwave expansion. These analyses have been performed for tightly bound projectiles $\left({ }^{10} \mathrm{Be}\right)$ from intermediate energies (67 MeV/nucleon) down to energies of interest of future RIB facilities such as HIE-ISOLDE and ReA12 at MSU (10 MeV/nucleon).

55th International Winter Meeting on Nuclear Physics

23-27 January 2017

Bormio, Italy

${ }^{*}$ Speaker. 


\section{Introduction}

The development of Radioactive-Ion Beams (RIB) has enabled the discovery of nuclei with very unexpected structures. In particular, in the neutron-rich region of the nuclear chart, halo nuclei have been observed. These exotic nuclei exhibit a very large matter radius due to the low binding energy of one or two neutrons, which allows them to decouple from the core of the nucleus and to form a diffuse halo [1]. They are modelled as two- or three-body objects: a compact core and one or two valence neutrons. As they are very short-lived, they cannot be studied through usual spectroscopic techniques but we can infer information about their structure from measurements of reaction processes coupled with an accurate model of the reaction $[2,3]$.

Nowadays some RIB facilities, like ISOLDE at CERN, provide exotic beams at $5 \mathrm{MeV} /$ nucleon and the goal is to reach $10 \mathrm{MeV} /$ nucleon. At such energies, precise models such as the ContinuumDiscretised Coupled Channel method (CDCC, see Refs. [2, 3, 4]) have large computational cost and can present convergence problems. The eikonal approximation is a quantal method which has a reduced computational time and can be easily interpreted. Unfortunately, it is valid only at high energies [5]. In this work, we investigate the extension of this model to lower energies through the study of two corrections.

These corrections have already given interesting results for different types of reaction at various energies. Indeed, the first correction, proposed by Wallace (see Refs. [6]), has also been analysed in Refs. $[7,8,9,10]$. Moreover, Refs. $[8,11,12]$ have demonstrated the efficiency of the second correction for Coulomb-dominated collisions. Because the latter correction works fine for collisions on heavy targets, we seek to extend it to nuclear dominated reactions, viz. light targets. In this work, we provide analyses of these corrections to simple cases, i.e. two-body collisions. Our final goal is to generalise them to three- and four-body collisions.

In Sec. 2, we describe the eikonal approximation for the elastic scattering and the two aforementioned corrections. Then, in Sec. 3, the numerical inputs used in our computations are given. From the analyses conducted on the differential cross sections for the elastic scattering of ${ }^{10} \mathrm{Be}$ off ${ }^{12} \mathrm{C}$, we conclude and propose an idea to pursue the extension of the eikonal model.

\section{Theoretical framework}

\subsection{The eikonal description of elastic scattering}

In this study, we consider the elastic scattering of a projectile $P$ of mass $m_{P}$ and charge $Z_{P} e$ impinging on a target $T$ of mass $m_{T}$ and charge $Z_{T} e$. We assume both nuclei to be structureless and spinless and their interaction to be simulated by a central optical potential $V$. Their relative motion can hence be described by the following Schrödinger equation

$$
\left[\frac{P^{2}}{2 \mu}+V(R)\right] \Psi(\mathbf{R})=E \Psi(\mathbf{R})
$$

where $\mathbf{R}$ is the projectile-target relative position, $\mathbf{P}$ the corresponding momentum, $\mu=m_{P} m_{T} /\left(m_{P}+\right.$ $\left.m_{T}\right)$ the $P-T$ reduced mass and $E$ the total energy of the system in the center-of-mass restframe.

To describe the aforementioned collision, Eq. (2.1) has to be solved with the condition that the projectile is impinging on the target with an initial momentum $\hbar \mathbf{K}=\hbar K \widehat{\mathbf{Z}}$, i.e., whose direction we 
choose for the $Z$-axis (see Fig. 1):

$$
\Psi(\mathbf{R}) \underset{Z \rightarrow-\infty}{\longrightarrow} e^{i K Z+\cdots}
$$

The "..." in this asymptotic expression indicates that the incoming plane wave is distorted by $V$, even at large distances.

The eikonal approximation assumes that at sufficiently high energy, the relative motion of the nuclei does not differ much from the initial plane wave of Eq. (2.2). It is then suggested to factorize that plane wave out of the wave function $\Psi[2,5,13]$

$$
\Psi(\mathbf{R})=e^{i K Z} \widehat{\Psi}(\mathbf{R}) .
$$

The new wave function $\widehat{\Psi}$, depending smoothly on $\mathbf{R}$, enables us to simplify the Schrödinger equation (2.1). Inserting Eq. (2.3) into Eq. (2.1) and neglecting the second-order derivative of $\widehat{\Psi}$ in comparison to its first-order derivative, leads to $[2,5,13]$

$$
i \hbar v \frac{\partial}{\partial Z} \widehat{\Psi}(b, Z)=V(b, Z) \widehat{\Psi}(b, Z),
$$

where $v=\hbar K / \mu$ is the initial velocity of the projectile relative to the target. In Eq. (2.4), we express explicitly the dependence of $\widehat{\Psi}$ on the transverse $\mathbf{b}$ and longitudinal $Z$ components of $\mathbf{R}$ as illustrated in Fig. 1.

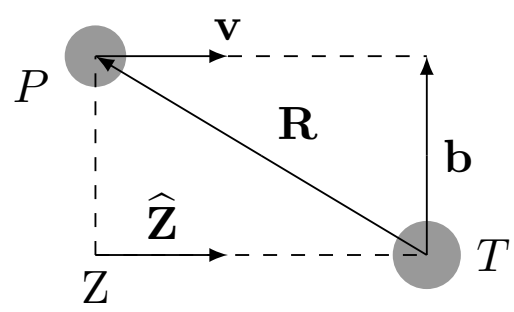

Figure 1: Coordinate system: the projectile-target relative coordinate $\mathbf{R}$ expanded in its transverse b and longitudinal $Z$ components.

The solutions of Eq. (2.4) read [2, 5, 13]

$$
\widehat{\Psi}(b, Z)=\exp \left[-\frac{i}{\hbar v} \int_{-\infty}^{Z} V\left(b, Z^{\prime}\right) \mathrm{d} Z^{\prime}\right] .
$$

This eikonal approximation of the wave function has a simple semiclassical interpretation: the projectile is seen as moving on a straight-line trajectory, accumulating a complex phase through its interaction with the target.

The scattering amplitude can be derived from these solutions as $[2,5,13]$

$$
f(\boldsymbol{\theta})=-\frac{i K}{2 \pi} \int \mathrm{d}^{2} b\left\{\exp \left[i \chi_{0}(b)\right]-1\right\} \exp (i \mathbf{q} \cdot \mathbf{b}),
$$

where $\hbar \mathbf{q}=\hbar \mathbf{K}^{\prime}-\hbar K \widehat{\mathbf{Z}}$ is the momentum transferred during the scattering process to reach the final momentum $\hbar \mathbf{K}^{\prime}$ and

$$
\chi_{0}(b)=-\frac{1}{\hbar v} \int_{-\infty}^{\infty} V(b, Z) d Z
$$


is the eikonal phase.

Since the eikonal phase Eq. (2.7) diverges for the Coulomb potential, that interaction requires a particular treatment. As indicated in Ref. [13], one should simply add to the eikonal phase computed with the nuclear part of the optical potential the Coulomb eikonal phase

$$
\chi^{C}=2 \eta \ln (K b)
$$

where $\eta=Z_{P} Z_{T} e^{2} / 4 \pi \varepsilon_{0} \hbar v$ is the Sommerfeld parameter. That phase leads to the exact Coulomb scattering amplitude.

At low energy, the eikonal approximation is no longer valid. However, since its implementation and interpretation are straightforward, it would be useful to extend its domain of validity to low energy. In the present work, we study two corrections which aim to take into account the deflection of the projectile by the target. The first one, proposed by Wallace (see Refs. [6]), acts on the nuclear interaction, while the second one can be applied to both interactions $[8,11]$.

\subsection{Wallace's correction}

It is derived from an expansion of the $T$-matrix whose exact form reads [6, 13]

$$
T=V+V G V,
$$

where $G$ is the exact propagator defined by $G^{-1}=E-P^{2} / 2 \mu-V+i \varepsilon$. This propagator can be expressed in terms of the eikonal propagator $g$ and a corrective term $N$ accounting for the deviations of the wave vector from the average wave vector $\widetilde{\mathbf{K}}=\left(\mathbf{K}^{\prime}+\mathbf{K}\right) / 2$ due to the $P-T$ interaction during the reaction process [6]

$$
G=g+g N G
$$

Wallace has obtained an expansion of the $T$-matrix by inserting iteratively this relation into Eq. (2.9)

$$
T=(V+V g V)+V g N g V+\ldots,
$$

where the terms in parenthesis correspond to the standard eikonal approximation.

In Refs. [6], it is shown that the scattering amplitude at the $m^{\text {th }}$ order can be expressed as

$$
f^{(m)}(\boldsymbol{\theta})=-\frac{i K}{2 \pi} \int \mathrm{d}^{2} b \mathscr{T}^{(m)}(b) \exp (i \mathbf{q} \cdot \mathbf{b}) .
$$

The zeroth order $\mathscr{T}^{(0)}$ corresponds to the standard eikonal model, developed by Glauber [5] [see Eq. (2.6)]. Wallace has derived the first three corrected orders of the $T$-matrices $\mathscr{T}^{(m)}$ in Refs. [6]. The first order reads

$$
\mathscr{T}^{(\mathrm{I})}(b)=\exp \left\{i\left[\chi_{0}(b)+\tau_{1}(b)\right]\right\}-1
$$

where $\tau_{1}$ is an additional phase depending on the derivative of the potential.

Two main limitations of this correction are identified in Refs. [6]. The expansion suggested by Wallace is valid only at sufficiently large energies and for potentials which vary smoothly. 


\begin{tabular}{lllll}
\hline \hline$V_{R}=123.0 \mathrm{MeV}$ & $W_{I}=65.0 \mathrm{MeV}$ & $R_{R}=3.33 \mathrm{fm}$ & $R_{I}=3.47 \mathrm{fm}$ & $a_{R}=a_{I}=0.80 \mathrm{fm}$ \\
\hline \hline
\end{tabular}

Table 1: Parameters of the potential used to simulate the ${ }^{10} \mathrm{Be}-{ }^{12} \mathrm{C}$ nuclear interaction [see Eq. (3.1)]. This potential is taken from Ref. [7].

These conditions ensure that the expansion parameter $\varepsilon$ and the derivatives of the potential take small values, which is necessary for the perturbation treatment to hold. Physically, it is due to the fact that, at lower energies, the $P-T$ relative motion differs more from the initial plane wave [see Eq. (2.3)]. We can also note that this correction is only significant for the nuclear interaction since the corrective terms vanish exactly for a potential decreasing as $1 / r$.

\subsection{Semi-classical correction}

As mentioned in Sec. 2.1, within the eikonal approximation framework, the projectile is seen as moving along straight-line trajectories and therefore the deflection of the projectile by the target is neglected. To improve the simulation of the Coulomb interaction within the eikonal model, we can replace the actual impact parameter $b$ in the eikonal phase by the distance of closest approach between the projectile and the target in the corresponding Coulomb trajectory [13, 14]. In Ref. [12], it was observed that this correction enables to account efficiently for that deflection in the Coulomb breakup of halo nuclei.

Similarly, an extension of this correction to the nuclear interaction is used in Refs. [8, 11]. To also account for the deflection due to the nuclear interaction, they have proposed to apply the same idea with the distance of closest approach between the two nuclei of the classical trajectory considering both interactions $[8,11]$. To ensure the conservation of the angular momentum, the eikonal phase is also multiplied by the ratio between the distance of closest approach and the actual impact parameter. It is equivalent to change the asymptotic velocity by the tangential velocity at the distance of closest approach. In this study, this distance is calculated from the real part of the potential, i.e. the Coulomb potential and the real part of the nuclear optical potential.

\section{Results}

\subsection{Projectile-target potentials}

To analyse the effects of the corrections presented in Sec. 2, we consider the elastic scattering of a nuclear-dominated reaction $\left({ }^{10} \mathrm{Be}\right.$ off $\left.{ }^{12} \mathrm{C}\right)$ at different energies. In this work, we use the potential developed in [7] to simulate the ${ }^{10} \mathrm{Be}-{ }^{12} \mathrm{C}$ interaction. The nuclear part is given by

$$
\begin{aligned}
& V_{N}(R)=-V_{R} f_{W S}\left(R, R_{R}, a_{R}\right)-i W_{I} f_{W S}\left(R, R_{I}, a_{I}\right), \\
& \text { with } \quad f_{W S}\left(R, R_{X}, a_{X}\right)=\frac{1}{1+e^{\frac{R-R_{X}}{a_{X}}}},
\end{aligned}
$$

with the different parameters listed in Table 1 . The Coulomb part of the interaction is simulated by the potential of a uniformly charged sphere of radius $R_{C}=1.2 \times\left(10^{1 / 3}+12^{1 / 3}\right) \mathrm{fm}$ [7]. Since the goal of the present study is to compare the standard eikonal model with its corrections, we use the same potential for all calculations and we neglect any energy dependence. 


\subsection{Analysis}

To evaluate the gain brought by each corrections presented in Sec. 2, we compare the cross sections for the elastic scattering and the $T$-matrices at two energies (67 and $10 \mathrm{MeV} /$ nucleon) with results obtained with a fully-converged partial-wave expansion, considered as exact. In Fig. 2, we represented these exact results by the red solid line and the standard eikonal model computations by the green dashed line.

As the terms of the second and the third orders of Wallace's correction are negligible, we only plot the first order in the blue short dashed line. This correction leads to nearly exact results at high energy (67 MeV/nucleon in Figs. 2(a) and 2(b)) but is less efficient at lower energy (10 MeV/nucleon in Figs. 2(c) and 2(d)) since there are still discrepancies with the exact results. Nevertheless, at low energy, the oscillation pattern of the cross sections is better reproduced than with the standard eikonal approximation. But the correction induces a shift of the cross sections to more forward angles and of the $T$-matrices to larger impact parameters. It can be explained by the attractive feature of the nuclear interaction: as Wallace's correction adds corrections to the nuclear phase, it increases the attractive force between the projectile and the target, leading to an underestimation of the scattering angle.

To counter this shift, the Coulomb repulsion needs to be better accounted for. This motivates the introduction of the semi-classical Coulomb correction, which leads to the results plotted in the magenta dotted line. At high energy, it has no impact and the good agreement is kept unchanged. At low energy, the semi-classical Coulomb correction compensates the shift induced by Wallace's correction and the calculations are now in phase with the exact ones. However, the resulting cross sections still lie above the exact ones at large angles. Therefore, we should enhance the absorption at small impact parameters to increase the accuracy.

This need for higher absorption as well as the desire to have only one consistent correction to both interactions has driven us to analyse the semi-classical correction applied to both interactions, corresponding to the black dotted line. At high energy, the accuracy of the eikonal model is worsened while at lower energies one can note a small improvement at forward angles (below $20^{\circ}$ ). It also reproduces well the oscillation pattern of the cross sections without inducing any shift in the results. Yet, even at low energy, this correction is still insufficient at large angles due to a lack of absorption.

\section{Conclusions}

In the present work, we have analysed two corrections and their interplay in a simple case, i.e. a two-body collision of light nuclei. Both corrections aim to account for the deflection of the projectile by the target due to both interactions, neglected in the standard eikonal model. To evaluate the accuracy gain brought by each corrections, we have computed the angular distribution of the cross sections for the elastic scattering of ${ }^{10} \mathrm{Be}$ off ${ }^{12} \mathrm{C}$ at two energies (67 and $10 \mathrm{MeV} /$ nucleon).

Results have shown that Wallace's correction is more efficient at high energies (67 MeV/nucleon) and reproduces well the oscillation pattern of the angular distribution. It also induces a shift to more forward angles at low energies (10 MeV/nucleon) which is cancelled when the semi-classical Coulomb correction is introduced [13, 14]. Both corrections combined enable an extension of the eikonal model to lower energies but the cross sections are still overestimated at large angles. 


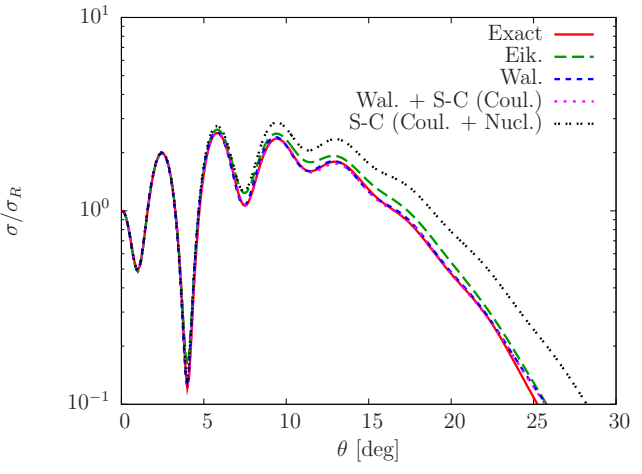

(a)

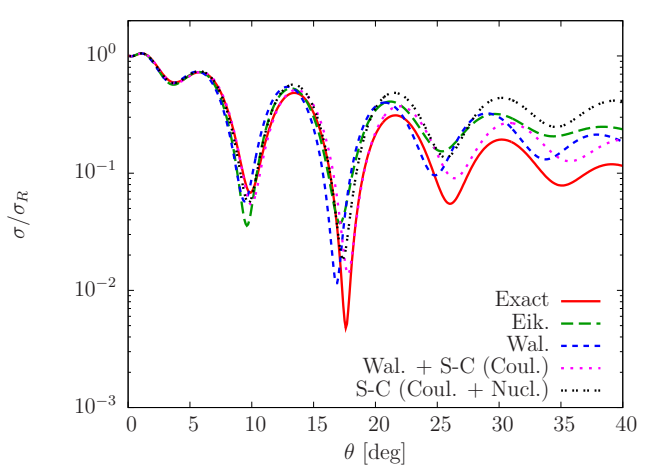

(c)

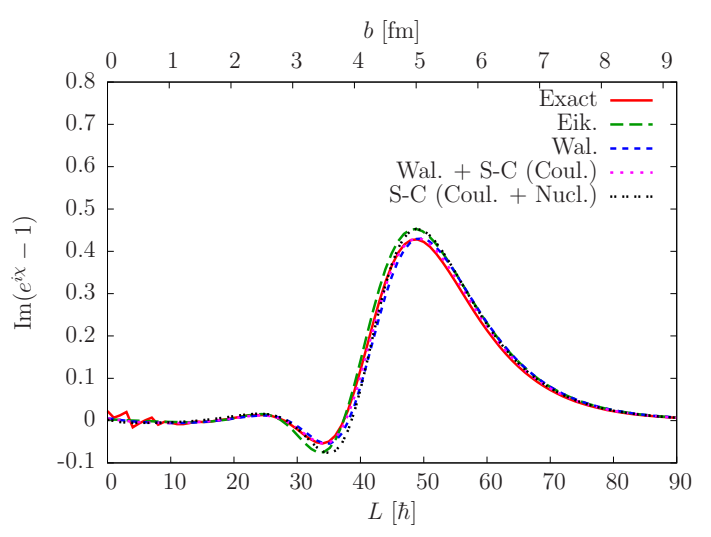

(b)

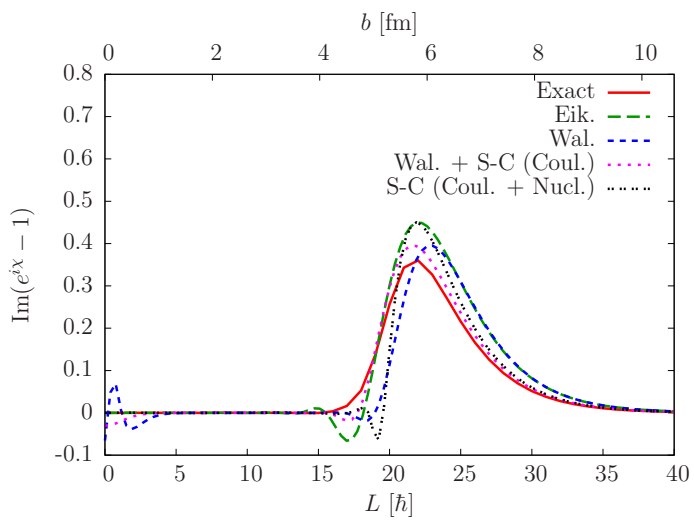

(d)

Figure 2: Elastic scattering of ${ }^{10} \mathrm{Be}$ off ${ }^{12} \mathrm{C}$ at $67 \mathrm{MeV} /$ nucleon $(\mathrm{a}, \mathrm{b})$ and $10 \mathrm{MeV} /$ nucleon $(\mathrm{c}, \mathrm{d})$. The cross sections are plotted as a ratio to Rutherford as a function of the scattering angle $\theta(\mathrm{a}, \mathrm{c})$ and the imaginary part of the $T$-matrices as a function of the angular momentum and the corresponding impact parameter $(b, d)$. The results are obtained with the partial-wave expansion (red solid line), the standard eikonal approximation (green long dashed line), Wallace's correction (blue short dashed line, see Sec. 2.2), Wallace's correction combined with the semi-classical Coulomb correction (magenta dotted line) and the semi-classical Coulomb and nuclear correction (black dotted line).

To cope with this inadequacy and to have one consistent correction, we have studied the semiclassical correction applied to both Coulomb and nuclear interactions $[8,11]$. The analysis has pointed out that there are no significant accuracy gain at low energies and that at high energies, the eikonal model leads to less precise results. The only improvement is the reproduction of the amplitude of the oscillations.

In conclusion, we have achieved an extension of the eikonal model to low energies but both corrections tested have failed at reproducing the absorption at large angles. To enhance absorption, we could apply the semi-classical correction with a complex distance of closest approach computed from the classical trajectory considering the whole optical potential [8]. In this way, the imaginary part of the potential would be increased for small impact parameters, hopefully this would cause a 
reduction of the cross sections at large angles.

\section{Acknowledgments}

C. Hebborn acknowledges the support of the Fund for Research Training in Industry and Agriculture (FRIA), Belgium. P. Capel acknowledges the support of the Deutsche Forschungsgesellschaft (DFG) with the Collaborative Research Center 1245. This work is part of the Belgian Research Initiative on eXotic nuclei (BriX), program P7/12 on inter-university attraction poles of the Belgian Federal Science Policy Office. This project has also received funding from the European Union's Horizon 2020 research and innovation program under grant agreement No 654002.

\section{References}

[1] I. Tanihata, J. Phys. G 22, 157 (1996).

[2] D. J. Baye and P. Capel, in Clusters in Nuclei (C. Beck (Springer), Berlin, 2012), vol. 2, p. 121.

[3] J. S. Al-Khalili and F. M. Nunes; J. Phys. G 29, R89 (2003).

[4] I. J. Thompson and F. M. Nunes. Nuclear reactions for astrophysics : Principles, calculation and applications of low-energy reactions. (Cambridge University Press, 2009).

[5] R. J. Glauber, in Lecture in Theoretical Physics, edited by W. E. Brittin and L. G. Dunham (Interscience, New York, 1959), vol. 1, p. 315.

[6] S. J. Wallace, Ph.D. thesis, University of Washington, Seattle (1971).

S. J. Wallace, Ann. Phys. 78, 190 (1972).

S. J. Wallace, Phys. Rev. Lett. 27, 622 (1971).

[7] J. S. Al-Khalili, J. A. Tostevin, and J. M. Brooke, Phys. Rev. C 55, R1018 (1997).

[8] C. E. Aguiar, F. Zardi and A. Vitturi, Phys. Rev. C 56, 1511 (1997).

[9] M. Buuck and G. A. Miller, Phys. Rev. C 90, 024606 (2014).

[10] B. V. Overmeire and J. Ryckebusch, Phys. Rev. Lett. B 650, 337 (2007).

[11] S. M. Lenzi, A. Vitturi and F. Zardi, Z. Phys. A 352, 303 (1995).

[12] T. Fukui, K. Ogata, and P. Capel, Phys. Rev. C 90, 034617 (2014).

[13] C. A. Bertulani and P. Danielewicz, Introduction to nuclear reactions (Institute of Physics Publishing, Bristol, 2004).

[14] R. A. Broglia and A. Winther, Heavy Ion Reactions, Lectures Notes, Vol. 1: Elastic and Inelastic Reactions (Benjamin-Cummings, Reading, England, 1981). 